Pesq. Vet. Bras. 30(8):651-658, agosto 2010

\title{
Avaliação radiográfica e ultrassonográfica do aparato podotroclear de cavalos Quarto de Milha diagnosticados com síndrome do navicular ${ }^{1}$
}

\author{
Cintia I.C. Peixoto*2, Luis C.Vulcano², Vânia M.V.Machado², Ana Liz G. Alves ${ }^{3}$ \\ e Ricardo H.T. Fanton ${ }^{4}$
}

\begin{abstract}
Peixoto C.I.C., Vulcano L.C., Machado V.M.V., Alves A.L.G \& Fanton R.H.T. 2010. [Radiographic and ultrasonographic evaluation of the podotrochlear apparatus in American Quarter horses diagnosed with navicular syndrome.] Avaliação radiográfica e ultrassonográfica do aparato podotroclear dos cavalos Quarto de Milha diagnosticados com a síndrome do navicular. Pesquisa Veterinária Brasileira 30(8):651-658. Departamento de Reprodução Animal e Radiologia Veterinária, Faculdade de Medicina Veterinária e Zootecnia, Universidade Estadual Paulista "Júlio de Mesquita Filho", Botucatu, SP 18618000, Brazil. E-mail: cintiamev@ hotmail.com

The objective of this study was to evaluate radiographically and ultrasonographically the podotrochlear apparatus using a transcuneal approach in 22 American Quarter horses suffering from navicular syndrome. On the radiographs, different forms of synovial invaginations, calcification of the podotrochlear bursa and deep digital flexor tendon, enthesophyte and osteophyte formation, and changes in the cortical-medullary margin were observed. On the ultrasound scans, changes of the podotrochlear bursa, adhesions and irregularities at the surface of the deep digital flexor tendon, the digital cushion area reduction, calcification of the impar distal sesamoideal ligament, and irregularities of the flexor surface of the distal sesamoid bone were observed. Radiographic and ultrasonographic abnormalities compatible with navicular syndrome were present in all patients. Ultrasonography using transcuneal approach was a practical and efficient method to evaluate podotrochlear apparatus injuries in equine, thus being a complementary method to radiography.
\end{abstract}

INDEX TERMS: Transcuneal ultrasound, navicular syndrome, lameness, podotrochlear apparatus.

RESUMO.- O objetivo deste estudo foi avaliar as alterações do aparato podotroclear de 22 cavalos da raça Quarto de Milha acometidos pela síndrome do navicular utilizando $o$ acesso transcuneal. Esses equinos foram submetidos ao exame radiográfico e ultrassonográfico. No exame radiográfico foi observado a presença de invaginação sinovial de diferentes tipos, além de calcificação da bursa

\footnotetext{
${ }^{1}$ Recebido em 2 de setembro de 2009.

Aceito para publicação em 6 de abril de 2010.

2 Departamento de Reprodução Animal e Radiologia Veterinária, Faculdade de Medicina Veterinária e Zootecnia (FMVZ), Universidade Estadual Paulista (Unesp), Botucatu, SP 18618-000, Brasil. *Autora para correspondência: cintiamev@ hotmail.com

${ }^{3}$ Departamento de Cirurgia e Anestesiologia Veterinária, FMVZ, Unesp-Botucatu, SP.

${ }^{4}$ Médico veterinário autônomo, Rodovia Marechal Rondon Km 338 , Rua Luiz Fernando Rocha e Coelho 9/105, Bauru, SP 17047-280, Brasil.
}

podotroclear e tendão flexor digital profundo, entesiófitos, osteófitos e alteração do limite córtico-medular. No exame ultrassonográfico observou-se alterações da bursa podotroclear, aderência e irregularidades nos bordos do tendão flexor digital profundo, diminuição do coxim digital, calcificação do ligamento sesamóide distal ímpar e irregularidade da superfície flexora do osso sesamóide distal. Todos os equinos que apresentavam alterações radiográficas também apresentaram alterações no exame ultrassonográfico compatíveis com a síndrome do navicular. O exame ultrassonográfico utilizando o acesso transcuneal foi um método prático e eficiente para avaliação das lesões do aparato podotroclear dos eqüinos, sendo um método complementar ao exame radiográfico.

TERMOS DE INDEXAÇÃO: Ultrassom transcuneal, síndrome do navicular, claudicação, aparato podotroclear. 


\section{INTRODUÇÃO}

As enfermidades locomotoras são as mais frequentes em eqüinos; dentre elas a síndrome do navicular que é uma enfermidade crônica e progressiva que afeta não apenas o osso sesamóide distal (OSD) ou osso navicular como também é conhecido, mas todo aparato podotroclear, sendo uma das causas de claudicação crônica nos equinos, que leva a redução do desempenho atlético gerando perdas econômicas no mercado eqüestre.

A síndrome do navicular é considerada como causa principal de um terço das claudicações nos cavalos de esporte (Turner 1990, Pleasant \& Crisman 1997), sendo específica dos equídeos (MacGregor 1989) e acomete cavalos entre 6 a 12 anos de idade sem distinção de sexo, caracterizando-se por uma claudicação intermitente, principalmente nos membros torácicos (Colles 1982). O diagnóstico, na maioria das vezes, baseia-se apenas nos sinais clínicos e na observação dos achados radiográficos. Buscando aperfeiçoar, as técnicas de diagnóstico por imagem nessa enfermidade, este estudo teve o intuito de avaliar radiograficamente e ultrassonograficamente as alterações presentes no aparato podotroclear dos eqüinos com a síndrome do navicular, utilizando especificamente o acesso transcuneal descrito por Busoni \& Denoix (2001).

\section{MATERIAL E MÉTODOS}

No presente estudo foram utilizados 22 equinos da raça Quarto de Milha sendo 8 fêmeas e 14 machos entre 3 a 20 anos de idade, competindo nas modalidades esportivas: laço, tambor e corrida. Todos os equinos do experimento já apresentavam diagnóstico clínico definido de síndrome do navicular feito pelos seus respectivos médicos veterinários e encaminhados para avaliação radiográfica e ultrassonográfica com acesso transcuneal. Para obtenção de imagens normais, foram utilizados 10 equinos machos e fêmeas, entre 3 e 20 anos, da raça Quarto de Milha sadios, da mesma modalidade esportiva, sem histórico clínico de claudicação, submetidos aos mesmos exames do grupo doente.

\section{Exame radiográfico}

Para a avaliação das alterações do osso sesamóide distal foram realizadas três projeções radiográficas em ambos os membros torácicos (direito e esquerdo) são elas: Lateromedial, Dorsoproximal-palmarodistal oblíqua de $60^{\circ}$ e Palmaroproximal-palmarodistal obliqua. Para o procedimento foram utilizados chassis $18 \times 24 \mathrm{~cm}$, Filme de raio $\mathrm{x}$ - verde, $18 \times 24 \mathrm{~cm}_{\text {Kodak }}{ }^{4}$ e um aparelho de raio-x portátil com 100mAs de potência, marca FNX-modelo jockey ${ }^{5}$. A kilovoltagem $(\mathrm{kVp})$ foi padronizada para $75 \mathrm{kVp}, 7 \mathrm{mAs}$ e a distância foco-filme de $60 \mathrm{~cm}$.

\footnotetext{
${ }^{4}$ Kodak® Medical X-ray Film General Purpose Green-MXG, Kodak do Brasil, Rod. Presidente Dutra Km154,7, Rio Comprido, São José dos Campos, SP 12240-420.

${ }^{5}$ FNX-90 Jockey®, Fábrica de Equipamentos Hospitalares Electra Ltda, Estrada do Quitungo 634, Brás de Pina, Rio de janeiro, RJ 21215563.

${ }^{6}$ Aparelho de ultrassom portátil, Honda, modelo HS-2000 ${ }^{\circledR}$. Echo P\&D, Rua Getúlio Vargas Filho 483, São Paulo, SP 04318-030.

${ }^{7}$ Carbogel $^{\circledR}$, Ind. e Comércio de Produtos Gelatinosos Ltda, Rua Itaipú 241, Saúde, SP 04143-010.
}

Antes do procedimento radiográfico, as ferraduras dos membros torácicos (direito e esquerdo) foram removidas, e os cascos foram lavados. O critério de avaliação das alterações radiográficas seguiu o padrão estabelecido por Colles (1982), nas seguintes projeções: lateromedial (presença de osteófito, entesiófito e radioluscência na superfície flexora); dorsoproximalpalmarodistal oblíqua (presença discreta $(+)$ invaginações sinoviais do tipo 1a e 2a, presença moderada (++) invaginações sinoviais do tipo 3a e 6a, grave (+++) para as lesões císticas); projeção palmaroproximal-palmarodistal oblíqua (desorganização da trabécula óssea, esclerose da cavidade medular, espessamento da córtex - flexor e zonas radioluscentes).

\section{Exame ultrassonográfico}

Os equinos foram submetidos ao exame ultrassonográfico utilizando o aparelho Honda HS- $2000^{6}$, em ambos os membros torácicos para avaliação da bursa podotroclear (BP), tendão flexor digital profundo (TFDP), ligamento sesamóide distal ímpar (LSDI), face flexora do osso sesamóide distal e coxim digital (CD). Antes de iniciar o procedimento a ranilha foi aparada e lavada com água e sabão. Após a limpeza do casco, este foi colocado dentro de uma bota de borracha contendo água no seu interior, por um período de 60 minutos. 0 exame foi feito com dígito em semi-flexão (Fig.1), com o transdutor linear $\mathrm{Honda}^{6}$ de $7,5 \mathrm{MHZ}$ posicionado na região central da ranilha, juntamente com o gel ${ }^{7}$ de transmissão. As imagens foram obtidas no plano sagital, e armazenadas. Todos os exames foram feitos com os equinos em estação e sem sedação.

O critério para avaliação das imagens ultrassonográficas seguiu o seguinte padrão: bursa podotroclear (espessura e ecogenicidade); tendão flexor digital profundo (espessura, ecogenicidade, integridade das margens dorsal e palmar); comprimento (palmarodorsal) do coxim digital; superfície flexora do osso sesamóide distal e ligamento sesamóide distal ímpar (ecogenicidade). As imagens ultrassonográficas das estruturas do aparato podotroclear foram comparadas com as imagens do estudo anatômico ultrassonográfico realizado por Sage \& Turner (2002).

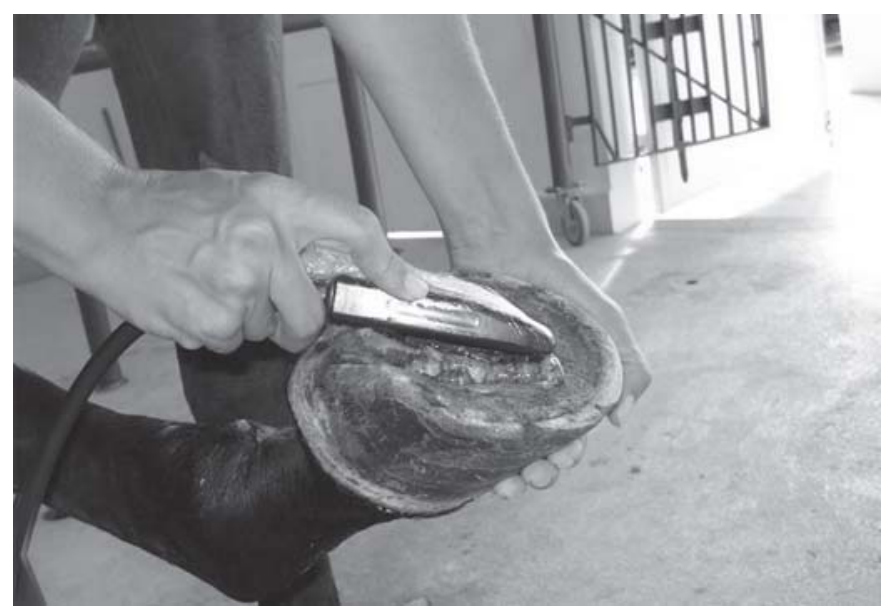

Fig.1. Exame ultrassonográfico utilizando o acesso transcuneal.

\section{RESULTADOS}

\section{Exame radiográfico}

Os resultados obtidos na avaliação radiográfica dos 22 equinos com a síndrome do navicular estão na Figura 2. 


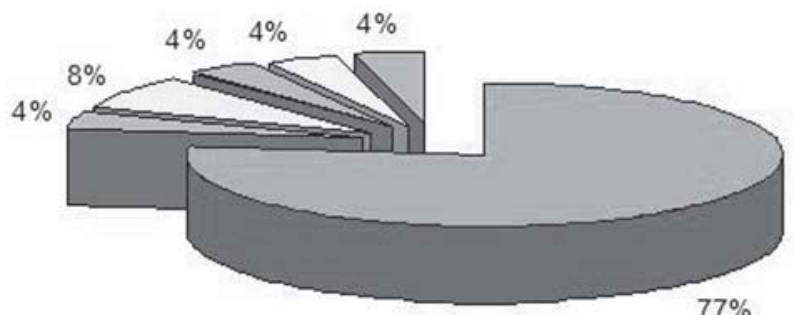

$\square$ Inva gina ção Sinovial
$\square$ Calcifica ção da Bursa Podotroclear
$\square$ Calcifica ção do TFDP
$\square$ Oste ófito
$\square$ Entesiófito
$\square$ Altera ção Córtico-Medular

Fig.2. Alterações do aparato podotroclear visualizadas no exame radiográfico dos equinos $(n=22)$ com síndrome do navicular.

Segundo os clínicos responsáveis, todos os equinos avaliados apresentavam sinais clínicos e alterações radiográficas compatíveis com síndrome do navicular. Vinte (77\%) dos equinos avaliados apresentaram invaginações sinoviais no bordo distal de diferentes tamanhos, formas e quantidades. Verifica-se na Figura 3 que as invaginações sinoviais apareceram com maior freqüência em ambos os membros (65\%), e, quando encontradas em um único

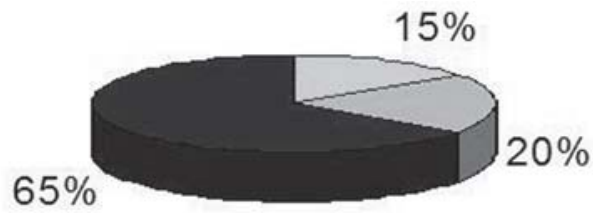

$\square M A D \quad \square M A E \quad \square M A / M A E$

Fig.3. Porcentagem da presença das invaginações sinoviais no bordo distal nos membros anteriores direito (MAD), membro anterior esquerdo (MAE) ou em ambos os membros (MAD/MAE) visualizadas no exame radiográfico dos equinos $(n=22)$ com a síndrome do navicular.

Quadro 1. Tipos de invaginações sinoviais classificadas como discreta (+), moderada (++) e grave (+++) e a porcentagem de acometimento dos MAD $^{\mathrm{a}}, \mathrm{MAE}^{\mathrm{b}}$ ou MAD/MAE

\begin{tabular}{lcccccc}
\hline & \multicolumn{7}{c}{ Tipos de invaginações sinoviais } \\
\cline { 2 - 7 } & MAD & $\%$ & MAE & $\%$ & MAD/MAE & $\%$ \\
\cline { 2 - 7 } Discreta (+) & 7 & $32 \%$ & 3 & $14 \%$ & 2 & $9 \%$ \\
Moderada (++) & 3 & $14 \%$ & 5 & $23 \%$ & - & $0 \%$ \\
Grave (+++) & 6 & $27 \%$ & 7 & $32 \%$ & 4 & $18 \%$
\end{tabular}

aMembro anterior direito; ${ }^{b}$ membro anterior esquerdo membro, o membro anterior esquerdo (MAE) $(20 \%)$ era mais acometido em relação ao membro anterior direito (MAD [15\%]) A avaliação da porcentagem das invaginações sinoviais presentes no bordo distal do osso sesamóide distal visualizadas no exame radiográfico com relação ao MAE, MAD ou ambos estão na Figura 3. Os tipos de invaginações sinoviais encontradas e caracterizadas como, discreta moderada e grave estão no Quadro 1.

\section{Quadro 2. Alterações da ecogenicidade da bursa podotroclear dos equinos $(n=22)$ com a síndrome do navicular}

\begin{tabular}{lccc}
\hline \multirow{2}{*}{ Alterações da ecogenicidade } & \multicolumn{3}{c}{ Número de membros afetados } \\
\cline { 2 - 4 } & MAD & MAE & MAD/MAE \\
\hline Hipoecóico & 12 & 15 & 8 \\
Anecóico & 7 & 15 & 3 \\
Não foi possível visualizar & 3 & - & -
\end{tabular}

aMembro anterior direito; ${ }^{\mathrm{b}}$ membro anterior esquerdo
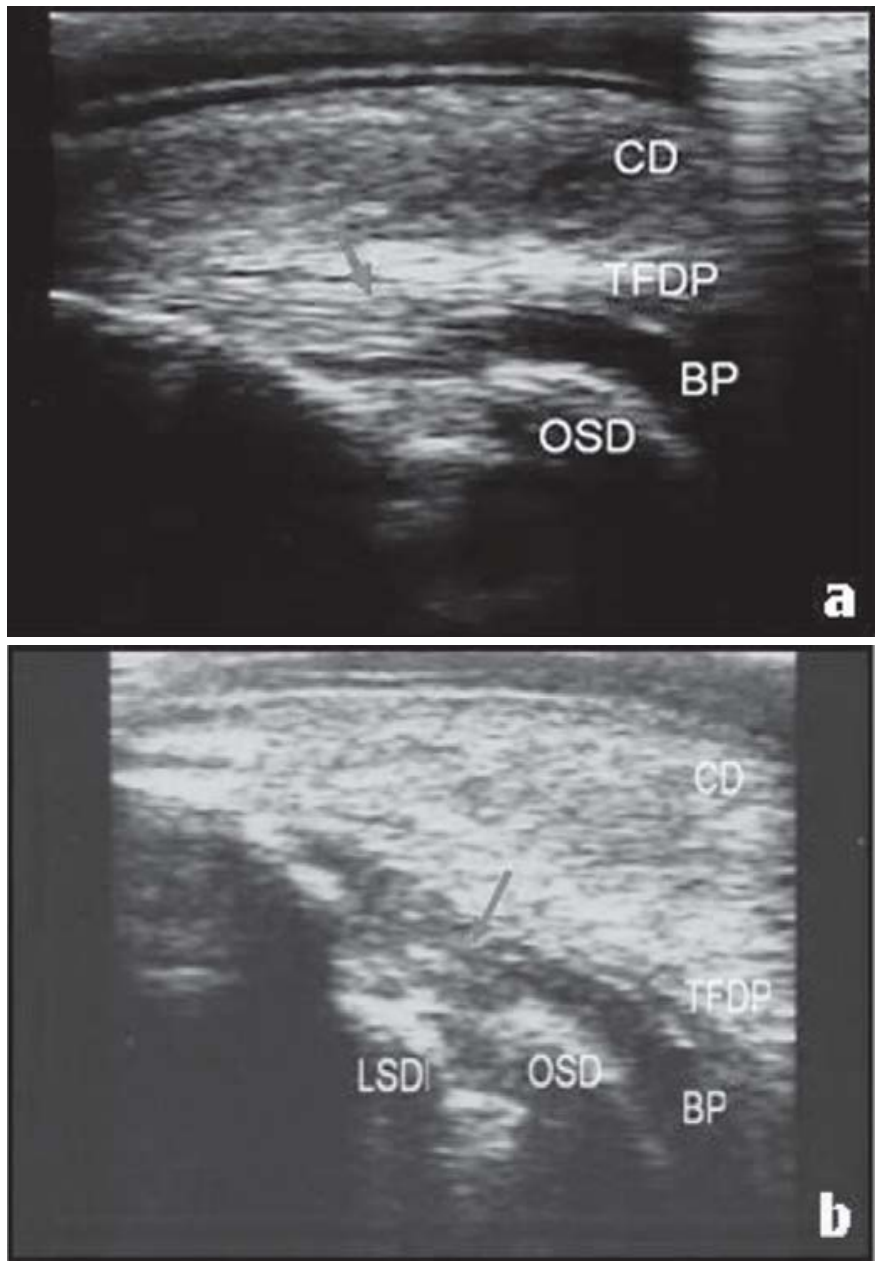

Fig.4. Imagens ultrassonográficas do aparato podotroclear dos equinos $(n=22)$ com a síndrome do navicular. (A) Área de aderência no tendão flexor digital profundo (TFDP) e grande quantidade pontilhado hiperecoico sugestivo de fibrina (seta) na bursa podotroclear (BP). Coxim digital (CD), osso sesamóide distal (OSD) (B) Irregularidade na margem palmar do TFDP (seta). 
As principais lesões encontradas no exame ultrassonográfico foram bursite, alterações tendíneas (aderências, perda de paralelismo das fibras e irregularidades das margens dorsal e palmar), calcificação do ligamento sesamóide distal ímpar, irregularidade da superfície flexora e alteração da espessura do coxim digital.

Bursa podotroclear. A avaliação ultrassonográfica da bursa podotroclear com relação a sua ecogenicidade esta demonstrada no Quadro 2 e as características da bursite aguda ou crônica estão demonstradas nas Figuras 4-7. A
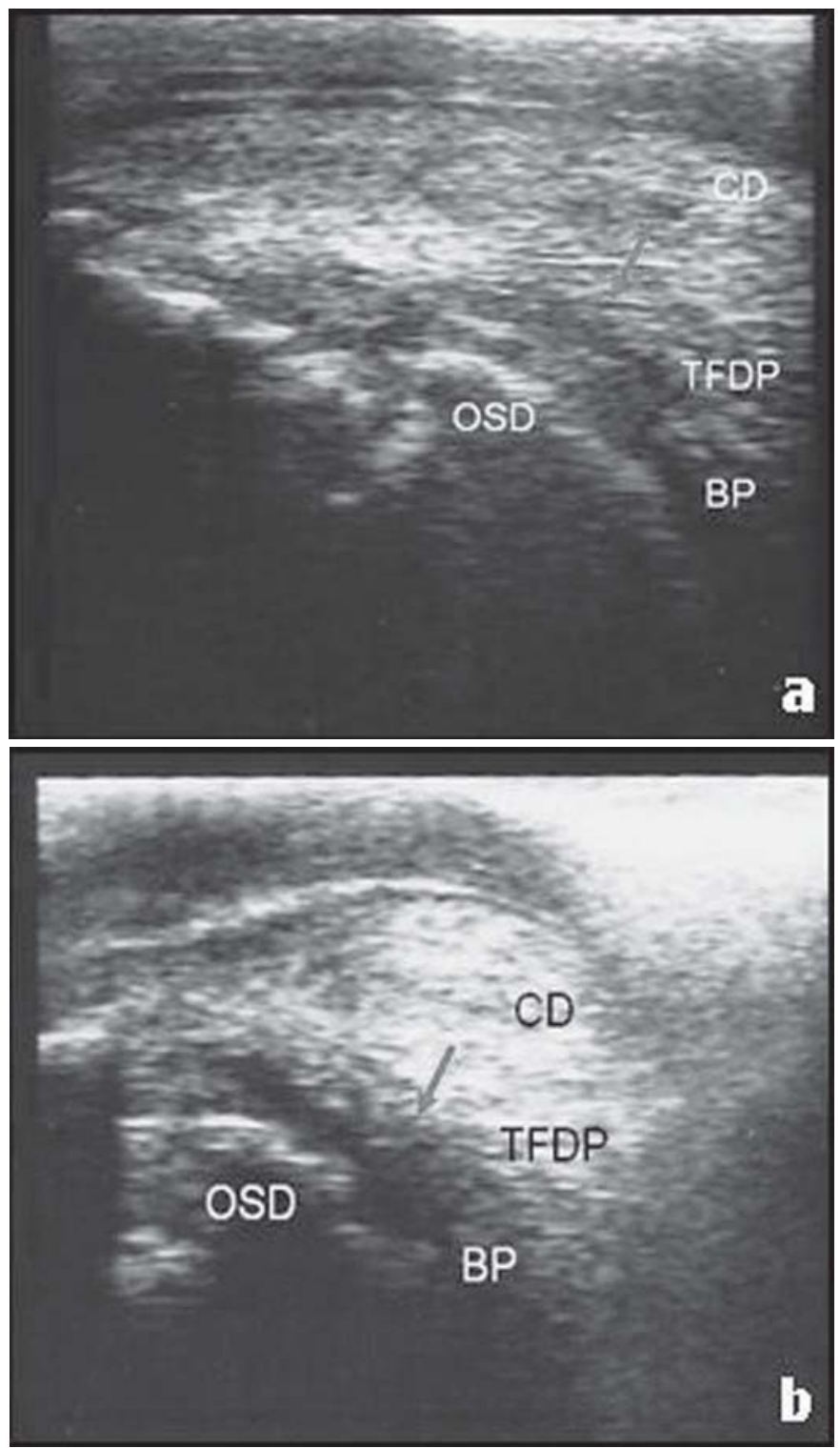

Fig.5. Imagens ultrassonográficas do aparato podotroclear dos equinos $(n=22)$ com a síndrome do navicular. (A) Irregularidade da margem dorsal do tendão flexor digital profundo (TFDP) com perda do paralelismo das fibras (seta). Coxim digital (CD). (B) Bursa podotroclear (BP) hipoecóica sugestiva de bursite aguda. Perda do paralelismo das fibras do TFDP e perda do limite das margens dorsal e palmar, irregularidade da superfície flexora do osso sesamóide distal (OSD).
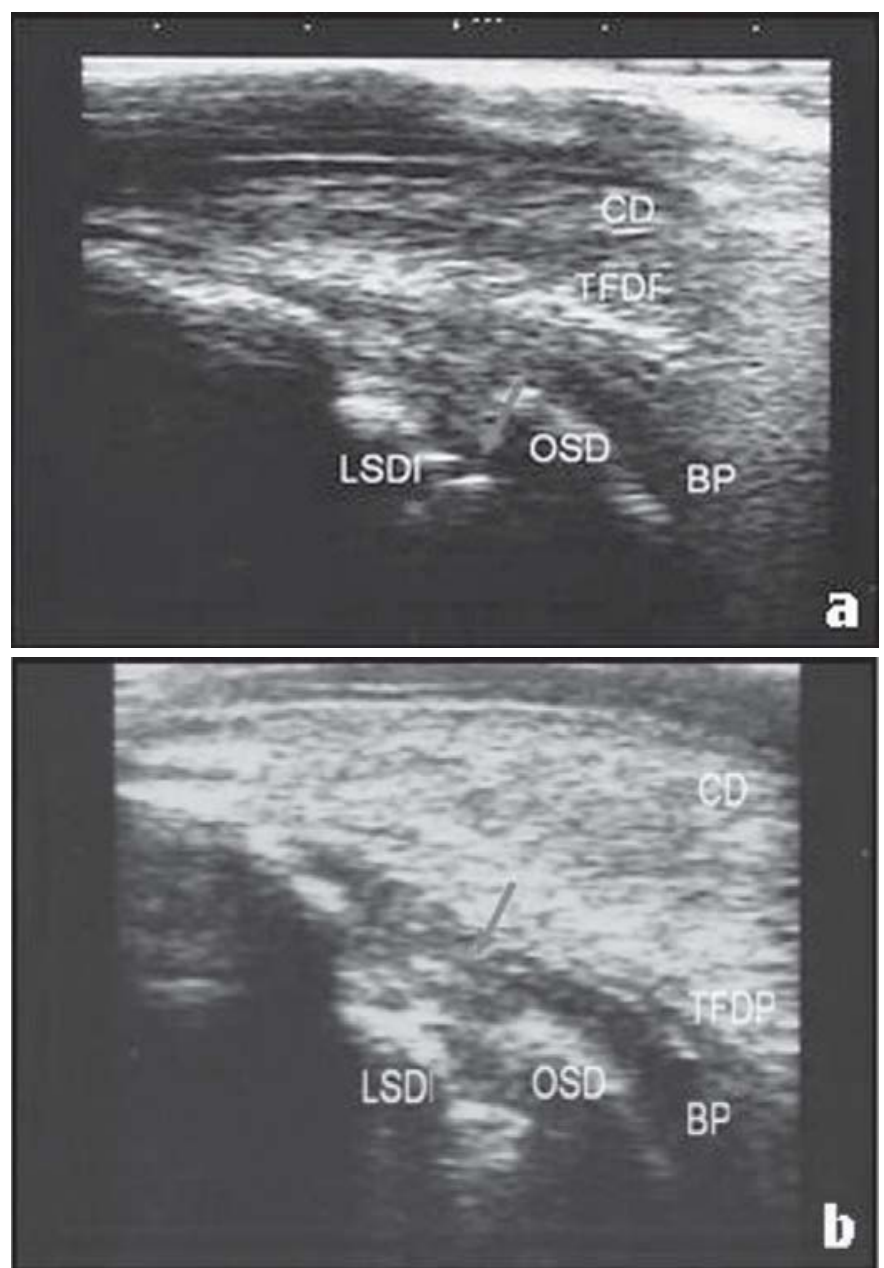

Fig.6. (A) Imagens ultrassonográficas da alteração da margem dorsal do tendão flexor digital profundo (TFDP) e calcificação do ligamento sesamóide distal ímpar (LSDI) (seta). Coxim digital (CD), osso sesamóide distal (OSD). (B) Pontilhado hiperecoico dentro da bursa podotroclear (BP) sugestivo de bursite crônica (seta).

mensuração pela ultrassonografia revelou uma média para MAD foi $3,26 \mathrm{~mm}$ e para MAE de $3,84 \mathrm{~mm}$. As imagens ultrassonográficas mostrando as alterações encontradas no aparato podotroclear dos equinos ( $n=22)$ com a síndrome do navicular estão apresentadas nas Figuras 4-7.

TFDP/ligamento anular. A média da mensuração do TFDP/Ligamento anular dos eqüinos avaliados $(n=22)$ encontrada no exame ultrassonográfico para MAD foi $3.93 \mathrm{~mm}$ e para MAE foi de $3,73 \mathrm{~mm}$. As alterações encontradas no TFDP/ligamento anular dos equinos avaliados $(n=22) \mathrm{com}$ a síndrome do navicular estão nas Figuras 4-6.

Coxim digital (CD). Os valores da mensuração ultrassonográfica do coxim digital dos equinos $(n=22)$ com a síndrome do navicular no MAD foi de $9,24 \mathrm{~mm}$ e para MAE foi de $9,25 \mathrm{~mm}$.

Ligamento sesamóide distal ímpar (LSDI). As alterações do ligamento sesamóide distal ímpar avaliado ultrassonograficamente dos equinos ( $n=22)$ com a síndrome do navicular relacionado aos números de membros afe- 

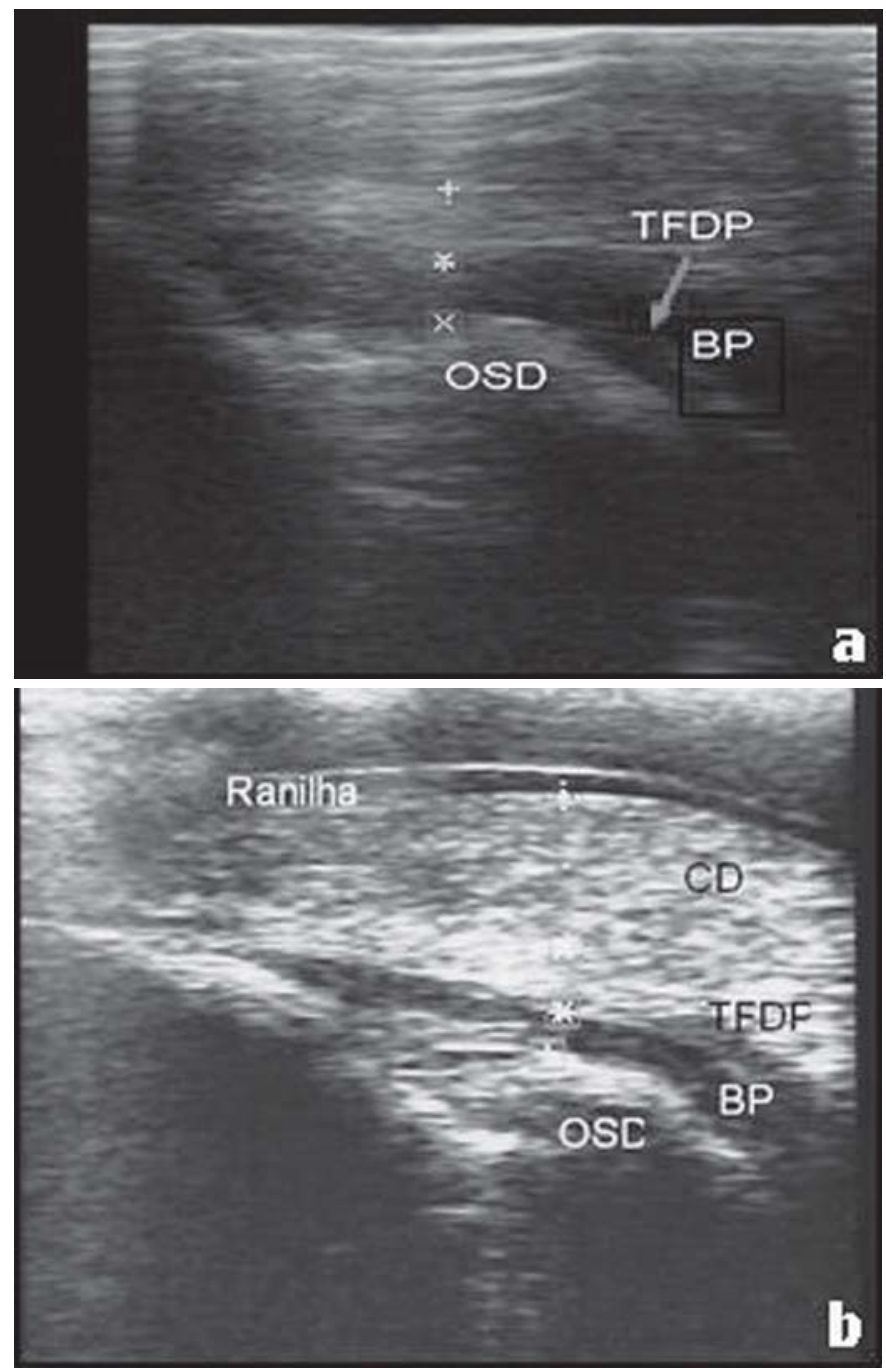

Fig.7. (A) Imagens ultrassonográficas do aumento da bursa podotroclear (BP) (seta) indicativo de bursite. Tendão flexor digital profundo (TFDP), osso sesamóide distal (OSD). (B) Imagem ultrassonográfica da diminuição da espessura do coxim digital (CD).

tados MAD, MAE ou MAD/MAE estão apresentados no Quadro 3.

Superfície flexora do osso sesamóide distal. As avaliações ultrassonográficas da superfície flexora do osso sesamóide distal (normal, irregular ou não foi possível visualizar) dos membros MAD, MAE e MAD/MAE dos equinos $(n=22)$ estão no Quadro 4.

Quadro 3. Alterações ultrassonográficas do ligamento sesamóide distal ímpar (LSDI)dos equinos $(\mathrm{n}=22) \mathrm{com}$ a síndrome do navicular

\begin{tabular}{lccc}
\hline \multirow{2}{*}{ Alterações do LSDI } & \multicolumn{3}{c}{ Número de membros afetados } \\
\cline { 2 - 4 } & MAD $^{\mathrm{a}}$ & MAE $^{\mathrm{b}}$ & MAD/MAE \\
\hline Normal & 4 & 2 & 9 \\
Com calcificação & 1 & 2 & 6 \\
Não foi possível visualizar & 2 & 3 & -
\end{tabular}

a Membro anterior direito; ${ }^{\mathrm{b}}$ membro anterior esquerdo.

\begin{tabular}{|c|c|c|c|}
\hline \multirow[t]{2}{*}{ Alterações da SF } & \multicolumn{3}{|c|}{ Número de membros afetados } \\
\hline & MAD & MAE & MAD/MAE \\
\hline Normal & 6 & 3 & 9 \\
\hline Superfície irregular & 3 & 5 & 2 \\
\hline Não foi possível visualizar & 2 & 3 & - \\
\hline
\end{tabular}

a Membro anterior direito; ${ }^{b}$ membro anterior esquerdo.

\section{Exame radiográfico}

\section{DISCUSSÃO}

O exame radiográfico foi de grande importância, pois serviu de referência para aplicação do exame ultrassonográfico transcuneal nos equinos com a síndrome do navicular, seguindo as orientações de Busoni \& Denoix (2001) e Grewal et al. (2004). Doige \& Hoffer (1983) observaram que essa enfermidade foi mais frequente nos equinos com a idade de 8-11 anos, contrastando com a idade dos equinos deste estudo. As alterações radiográficas encontradas nos eqüinos avaliados nesse estudo foram mais freqüentes em ambos os membros (MAD/MAE) do que apenas em um membro (MAD ou MAE), de acordo com os achados de Ackerman et al. (1977).

Nos eqüinos avaliados neste estudo, as alterações radiográficas predominantes no osso sesamóide distal (Fig.3) foram as invaginações sinoviais no bordo distal. Achado semelhante foi relatado por Ackerman et al. (1977), Colles (1979, 1982), MacGregor (1986), Pool (1995) e Ruohoniemi et al. (1998). Entretanto, Kaser-Hotz \& Ueltschi (1992) relatam que o aparecimento dessas invaginações sinoviais no bordo distal pode não ser conclusiva para o diagnóstico da síndrome do navicular. Colles (1979) e Rijkenhuizen et al. (1989), acreditam que a presença dessas alterações, invaginações sinoviais, estejam relacionadas com o suprimento sangüíneo reduzido, levando a degeneração do osso. Estudos de Poulos (1983) descrevem que as invaginações sinoviais presentes no bordo distal possivelmente sejam resultante da compressão causada pela bursa podotroclear ou tendão flexor digital profundo sobre essa região. Wright \& Douglas (1993) e Pool (1995) por reconhecer como coerente a afirmação de Poulos (1983) sobre a compressão causada pela bursa e pelo tendão flexor digital profundo, afirmam existir correlação com a conformação da região da quartela e os desvios dos eixos podofalângicos.

Na Figura 3 pode-se observar que as invaginações sinoviais foram as lesões mais freqüentes observadas em ambos os membros direito e esquerdo (65\%) dos equinos, sendo compatível com os achados de Ackerman et al. (1977) que citam que essas alterações foram bilaterais e semelhantes. Ao contrário da citação de Kaser-Hotz \& Ueltschi (1992) que observou que essas lesões foram mais freqüentes no MAD (55\%).

Os dados apresentados no Quadro 1 relacionam os tipos de invaginações sinoviais mais freqüentes presentes 
no bordo distal do OSD dos equinos com a síndrome do navicular, confirmando a citação de Colles (1982) que diz que esses tipos de invaginações são sugestivos de síndrome do navicular. Observou-se um membro com calcificação da BP (Fig.2) no exame radiográfico, porém não há na literatura citações desse tipo de alteração vista ao exame radiográfico. A calcificação do TFDP (Fig.2) ou mineralização distrófica do TFDP como também é conhecida, ocorreu apenas em dois membros e segundo Butler et al. (1993) a causa desse foco de mineralização proximal ao osso sesamóide distal é desconhecida, porém pode estar associada à processo inflamatório. Ao contrário da citação de O'Brien (2006) que justifica o aparecimento dessa alteração como causa iatrogênica devido a múltiplas aplicações de corticosteróides local na região da bursa ocasionando a calcificação do tendão flexor digital profundo e do coxim digital.

Com relação à presença de osteófito, observou-se apenas em um membro, reforçando a afirmação de Doige \& Hoffer (1983) que não associam essa alteração isolada com equinos com histórico de síndrome do navicular e sim como uma alteração que aparece em equino senil. Segundo Pottenger \& Phillips (1990) e Stashak (1994) a presença de osteófitos está relacionada com defeitos de conformação dos membros. Por outro lado, Dyson \& Kidd (1993) e Turner \& Anderson (1993) relatam que o aparecimento de osteófito pode estar associado à enfermidade articular degenerativa da articulação interfalangeana distal.

Neste estudo observou-se que apenas um membro dos equinos avaliados apresentou entesiófito (Fig.2), o que apóia a citação de Kaser-Hotz \& Ueltschi (1992) que este achado isolado não confere sinal radiográfico indicativo da síndrome do navicular, pois pode ser encontrado inclusive em eqüinos clinicamente sadios. Na opinião de Dyson (1988), com exceção daqueles casos em que ocorra concomitantemente a calcificação de tecidos moles, este é um achado radiográfico de significado clínico duvidoso. Park (1989), Butler et al. (1993) e Ruohoniemi et al. (1998) só consideram esse achado de importância clínica quando aparece na origem do LSDI.

A alteração do limite córtico-medular foi observada somente em um equino. Butler et al. (1993), Hernández \& Novales (1999) e Clercq et al. (2000) citam que essa alteração está relacionada com processo de remodelação e reabsorção óssea. Já Pool (1995) classifica esse achado indicativo de uma lesão crônica do osso sesamóide distal. Os resultados do estudo sugerem que a formação do tipo de lesões radiográficas no osso sesamóideo distal dos equinos avaliados pode ter correlação com tipo de atividade esportiva que esses equinos desempenham, concordando com a suspeita de Wintzer (1990). Observou-se também diferença na arquitetura ou forma anatômica do bordo proximal do osso sesamóide distal dos equinos avaliados nesse experimento com síndrome do navicular como afirmam Dik \& Van Den Broek (1995) e Rijkenhuizen (2006). Ruohoniemi et al. (1998), Dik et al. (2001) e Rijkenhuizen (2006) descrevem que o formato do bordo proximal é geneticamente determinado e podem contribuir como fator predisponente para o desenvolvimento da síndrome do navicular.

Exame ultrassonográfico. $\mathrm{O}$ exame ultrassonográfico utilizando o acesso transcuneal foi de grande importância nesse estudo, pois forneceu informações das alterações do aparato podotroclear associada à síndrome do navicular de acordo com as citações Busoni \& Denoix (2001), Busoni et al. (2002), Sage \& Turner (2002), Kristoffersen \& Thoefner (2003) e Grewal et al. (2004). O exame ultrassonográfico vem sendo utilizado principalmente para o diagnóstico de lesões nos tecidos moles do aparelho locomotor dos eqüinos, no entanto a prática deste exame é recente com acesso transcuneal (Kristoffersen \& Thoefner 2003; Grewal et al., 2004). Para avaliação das estruturas do aparato podotroclear utilizou-se um transdutor linear de $7,5 \mathrm{MHz}$ que proporcionou imagens de boa qualidade. Já Sage \& Turner (2002) também utilizaram o mesmo transdutor, porém com a freqüência de $10 \mathrm{MHz}$, diferentemente do estudo de Busoni \& Denoix (2001) e Kristoffersen \& Thoefner (2003) que utilizaram um transdutor setorial de 7,5 MHZ. Já Busoni et al. $(2002,2006)$ utilizaram simultaneamente transdutores linear e microconvexo de $7,5 \mathrm{MHz}$ e Grewal et al. (2004) utilizaram os mesmos tipos de transdutores, porém a freqüência foi $6,5 \mathrm{MHz}$. Utilizandose a técnica de pedelúvio durante 60 minutos para umedecer a ranilha que funciona como janela acústica sendo importante para a obtenção das imagens ultrassonográficas. Kristoffersen \& Thoefner (2003) descrevem que o casco deve permanecer no pedelúvio durante 1-3 horas. Grewal et al. (2004) diverge a afirmação e descreve que o casco deve permanecer de molho durante 12 horas para a obtenção de uma imagem de qualidade.

Considerando os resultados obtidos no exame ultrassonográfico da bursa podotroclear dos equinos avaliados, demonstrado no Quadro 2, observamos que dos 44 membros (direito e esquerdo) avaliados, 15 (68\%) membros esquerdos apresentaram a bursa podotroclear hipoecóica sendo sugestiva de bursite aguda (Fig.5 e 7). Estes resultados se assemelham com os descritos por Grewal et al. (2004) que também observou a alteração da ecogenicidade e quantidade do fluido sinovial compatível com o quadro de bursite aguda, descrito nos equinos com síndrome do navicular.

Observou-se que o membro anterior (MAE) esquerdo foi o que mais apresentou pontilhados hiperecoicos que são descritos por Grewal et al. (2004) como achados característicos de bursite crônica. O estudo da mensuração da bursa podotroclear pelo ultrassom ressalta que a média dos valores da mensuração da bursa podotroclear difere dos valores encontrados por Grewal et al. (2004) que foi de 2,03 $\mathrm{mm}$ para espessura bursa podotroclear.

Os resultados obtidos referentes às tendopatias do TFDP/ligamento anular demonstraram que a maioria apresentou irregularidades das margens dorsais (Fig.5 e 6) e palmar (Fig.4) do tendão, e perda de paralelismo das fibras tendíneas (Fig.5) e áreas com grandes aderências e fibrose (Fig.4). Adicionalmente, esses resultados reforçam os achados descritos por Grewal et al. (2004) que além dessas alte- 
rações também observaram casos de tendinite e mineralização distrófica dentro do TFDP, sendo considerada por Busoni et al. (2006) como uma alteração característica de tendinite crônica. Pode-se complementar ainda que as alterações acima descritas também apóiam as afirmações de Kristoffersen \& Thoefner (2003), os quais descrevem que essas áreas de aderências são encontradas próximas à superfície flexora do osso sesamóide distal, levando ao aparecimento de dor palmar. Ao contrário dos achados de Kristoffersen \& Thoefner (2003) que observaram essas áreas de aderência no tendão flexor digital profundo na sua porção mais distal e próximo a área do ligamento sesamóide distal ímpar e inserção com a falange distal (Fig.4).

Os resultados obtidos da média de mensuração dos TFDP/ligamento anular não foram compatíveis com o padrão de mensuração estabelecido por Grewal et al. (2004), pois os equinos avaliados por eles eram de raças diferentes, não representando uma amostra homogênea para padronização. A média da mensuração dos valores do coxim digital dos equinos avaliados não foi compatível com a média de 12,64mm encontrada por Grewal et al. (2004). No estudo foi possível observar as alterações do aparato podotroclear utilizando o acesso transcuneal, porém em dois equinos não foi possível visualizar as estruturas do aparato podotroclear via acesso transcuneal, sendo que os mesmos foram submetidos a protocolo semelhante e apresentavam ranilha de boa qualidade. Possivelmente esses equinos poderiam ter algum problema no coxim digital que não permitiu a passagem das ondas sonoras. O'Brien (2006) descreve que alguns equinos apresentam calcificação do coxim digital de forma iatrogênica, devido a múltiplas aplicações de corticosteróides local na região da bursa podotroclear, o que pode ser correlacionado com os equinos descritos acima.

Observou-se que na avaliação do ligamento sesamóide distal ímpar, um total de 9 dígitos (MAD, MAE e MAD/ MAE) apresentaram pontos de calcificação (Quadro 3, Fig. 6). Grewal et al. (2004) também descreveram o aparecimento da calcificação do LSDI. Segundo Butler et al. (1993) a calcificação é decorrente das constantes forças de tensões no ligamento. Blunden et al. (2006) descreve que a dor palmar nos equinos com síndrome do navicular também está relacionada a alteração do LSDI, pois no interior desse ligamento existe uma rede de inervação o que justificaria a dor palmar contínua. Um total de 10 dígitos (Quadro 4) (MAD e MAE) apresentaram irregularidades na superfície flexora (Fig.5) no exame ultrassonográfico. Kristoffersen \& Thoefner (2003), Grewal et al. (2004) e Busoni et al. (2006) também encontraram anormalidades da superfície flexora. Kristoffersen \& Thoefner (2003), afirmam que essas irregularidades da superfície flexora estão relacionadas com áreas de erosão na fibrocartilagem, sendo uma alteração comumente observada nos equinos com esta síndrome. Nesse estudo, não foi possível identificar as alterações da superfície flexora no exame radiográfico, pois foram observadas evidências somente no exame ultrassonográfico. Segundo Grewal et al. (2004) e Busoni et al.
(2006) o exame com o ultrassom mostra-se na maioria das vezes mais sensível às alterações da superfície flexora do que o exame radiográfico convencional. Todos os eqüinos avaliados apresentaram alteração no aparato podotroclear compatíveis com as alterações relatadas na síndrome do navicular. É importante salientar que a qualidade das imagens nem sempre foi satisfatória principalmente quando a ranilha não apresentava boa qualidade.

Portanto, o exame ultrassonográfico com acesso transcuneal deve ser utilizado na rotina, para detecção das injúrias do aparato podotroclear associado ao exame radiográfico (Grewal et al. 2004). Durante este estudo foram avaliados 10 equinos normais sem histórico clínico da síndrome navicular, porém submetidos ao mesmo tipo de treinamento. Esses equinos não mostravam sinais clínicos e nem alteração radiográfica no osso sesamóide distal compatível com a síndrome do navicular. Entretanto, apresentaram alterações ultrassonográficas no aparato podotroclear compatível com a síndrome do navicular. Esse fato sugere que a enfermidade apresenta-se também de forma sub-clínica e que o exame ultrassonográfico contribuiria para a detecção precoce da síndrome no seu estágio inicial. Considerando os diferentes aspectos estudados, salienta-se a necessidade de estudos para avaliar o aparato podotroclear e estabelecer um padrão de mensuração na raça Quarto de Milha, já que essa enfermidade é muito freqüente nos eqüinos dessa raça, como afirma a citação Ackerman et al. (1977).

\section{CONCLUSÕES}

A análise dos resultados obtidos, nas condições em que foi realizado o experimento, permite as seguintes conclusões:

O acesso transcuneal é um método prático e eficiente para avaliação das lesões do aparato podotroclear dos eqüinos sendo um método complementar ao exame radiográfico fornecendo informações adicionais que não são detectáveis pelo exame radiográfico.

O transdutor linear com freqüência de $7,5 \mathrm{MHz}$ foi adequado para avaliação das estruturas do aparato podotroclear e o tempo preconizado nesse estudo de 60 minutos no pedelúvio foi suficiente para a obtenção das imagens de boa qualidade.

Existe a necessidade de um estudo para estabelecermos o padrão de mensuração das estruturas do aparato podotroclear dos equinos da raça Quarto de Milha e das diferentes raças.

\section{REFERÊNCIAS}

Ackerman N., Johnson H.J. \& Dorn R. 1977. Navicular disease in the horse: risck factors radiographic changes and response to therapy. J. Am. Vet. Med. Assoc. 170(2):183-187.

Blunden A., Dyson S., Murray R. \& Schrame M. 2006. Histopathology in horses with chronic palmar foot pain anda ge-matched control. Part 1. Navicular bone and related stuctures. Equine Vet. J. 38:1522.

Butler J.A, Colles C.M., Dyson S.J., Kold E.S. \& Poulos W.P. 1993.Clinical 
Radiology of the Horse. Blackwell Scientific Publication, Oxford, p.5371.

Busoni V. \& Denoix J.M. 2001. Ultrasonography of the podotrochlear apparatus in the horse using a transcuneal approach: Technique and reference images. Vet. Radiol. Ultrasound 42:534-540.

Busoni V., Mean M.N., Brignone L. \& Snaps F. 2002. Échographie de l'appareil podo trochléaire: étude in vitro sur 30 membres isolés de cheval. Ann. Med. Vét. 146:181-187.

Busoni V., Lahaye B. \& Denoix J.M. 2006. Transcuneal ultrasonographic findings in the podotrochlear apparatus: Comparison with postmortem in 14 equine digits. J. Equine Vet. Sci. 26:113-117.

Clercq T., Verschooten F. \& Ysebaert M.A. 2000. Comparision of the palmaroproximal-palmarodistal view of the isolated navicular bone to otheir views. Vet. Radiol. Ultrasound 41:525-533.

Colles C.M. 1979. Ischaemic necroses of the navicular bone and its treatment. Vet. Rec. 17:133-137.

Colles C.M. 1982. Navicular disease and its treatment. In Practice 4:29-36.

Dik K.J. \& Van den Broek J. 1995. Role of navicular bone shape in the pathogenesis of navicular disease: A radiological study. Equine Vet. J. 27:390-393.

Dik J.K., Van den Belt M.J.A. \& Enzerink V.W.R.P. 2001. The radiographic development of the distal and proximal double contours of the equine navicular bone on dorsoproximal-palmarodistal oblique (upright pedal) radiographs, from age 1 to 11 months. Equine Vet. J. 33:70-74

Doige C.E. \& Hoffer M.A. 1983. Pathological changes in the navicular bone and associated structures of the horse. Can. J. Comp. Med. 47:387-395.

Dyson S. 1988. Variations in the normal radiographic anatomy of equine limbs. In Practice 10:119-125.

Dyson S.J. \& Kidd L. 1993. A comparasion of responses to analgesia of navicular bursa and intra-articular analgesia of distal interphalangeal joint in 59 horses. Equine Vet. J. 25:93-98.

Grewal S.J., McClure R.S., Booth L., Evans B.R. \& Caston S.S. 2004. Assessment of the ultrasonographic characteristics of the podotroclear apparatus in clinically normal horses and horses with navicular syndrome. J. Am. Vet. Med. Assoc. 225:1881-1888.

Hernández E.M. \& Novales M. 1999. Diagnóstico radiológico del síndrome podotroclear. Imag. Vet. 2:66-68.

Kaser-hotz B. \& Ueltschi G. 1992. Radiographic appearance of navicular bone in sound horses. Vet. Radiol. Ultrasound 33:9-17.

Kristoffersen M. \& Thoefner B.M. 2003. Ultrasonography of the navicular region in horses. Equine Vet Educ. 15:150-157.
MacGregor C.M. 1986. Radiographic assessment of navicular bones, based on changes in the distal nutrient foramina. Equine Vet. J. 18:203-206.

MacGregor C.M. 1989. Navicular disease: In search of definition. Equine Vet. J. 21:389-391.

O’Brien R.T. 2006. Interpretação radiográfica do osso navicular, p.92113. In: Ibid. (Ed.), Radiologia de Eqüinos. Roca, São Paulo.

Park D.R. 1989. Radiographic examination of the equine foot. Vet. Clin. North Am., Equine Pract. 5:47-66.

Pleasant S.R. \& Crisman M.V. 1997. Navicular disease in horses: Pathogenesis and diagnosis. Vet. Med. Comp. Anim. Pract. 92:250257.

Pool R.R. 1995. Pathophysiology of navicular disease. Proc. American Equine Sport Medicine, Davis, p.4-44.

Poulos P.W. 1983. Correlation of the radiographic signs and histologic changes in navicular disease. Proc. Annu. $29^{\text {th }}$ Conv. Am. Assoc. Equine. Pract., Las Vegas, p.241-255.

Pottenger L.A. \& Phillips F.M. 1990. The effect of marginal osteophytes on reduction of varus-valgus instability in osteoarthritic knees. Arthritis Rheum. 33:853-858.

Rijkenhuizen M.B.A., Nemeth F., Dik J.K. \& Goedegebuure A.S. 1989b. The arterial supply of the navicular bone in adult horses with navicular disease. Equine Vet. J. 21:418-424.

Rijkenhuizen M.B.A. 2006. Navicular disease: A review of what's new. Equine Vet. J. 38:82-88.

Ruohoniemi M., Ryhane V. \& Tulamo M.R. 1998. Radiographic appearance of the navicular bone and distal interphalangeal joint and their relationship with ossification of the collateral cartilages of the distal phalanx in finnhorse cadaver forefeet. Vet. Radiol. Ultrasound 39:125-132.

Sage M.A. \& Turner A.T. 2002. Ultrasonography of the soft tissue structures of the equine foot. Equine Vet. Educ. 14:221-224.

Stashak T.S. 1994. Claudicação em eqüinos segundo Adams, p.517532. In: Ibid. (Ed.), Claudicação. Roca, São Paulo.

Turner A.T. 1990. Navicular disease, p.413-416. In: White N. \& Moore N.J. (Eds), Current Practice of Equine Surgery. W.B. Saunders, Philadelphia.

Turner A.T. \& Anderson B. 1993. Lameness of the distal interphalangeal joint. Proc. $39^{\text {th }}$ Annual Convention of the American Association of Equine Practitioners, San Antonio, Texas, p.252-257.

Wintzer H.J. 1990. Doenças dos Eqüinos. Manole, São Paulo, p.226228.

Wright I.M. \& Douglas J. 1993. Biomechanical considerations in the treatment of navicular disease. Vet. Rec. 133:109-114. 